\title{
Circuit
}

Musiques contemporaines

\section{$32{ }^{\mathrm{e}}$ Festival International du Film sur l'Art (20-30 mars 2014)}

\section{Anne Marie Messier}

Volume 24, numéro 2, 2014

URI : https://id.erudit.org/iderudit/1026187ar

DOI : https://doi.org/10.7202/1026187ar

Aller au sommaire du numéro

Éditeur(s)

Les Presses de l’Université de Montréal

ISSN

1183-1693 (imprimé)

1488-9692 (numérique)

Découvrir la revue

Citer ce compte rendu

Messier, A. M. (2014). Compte rendu de [ $32^{\mathrm{e}}$ Festival International du Film sur l'Art (20-30 mars 2014)]. Circuit, 24(2), 93-96. https://doi.org/10.7202/1026187ar d'utilisation que vous pouvez consulter en ligne.

https://apropos.erudit.org/fr/usagers/politique-dutilisation/ 


\section{$32^{\mathrm{e}}$ Festival International du Film sur l'Art (20-30 mars 2014)}

Compte rendu d'Anne Marie Messier

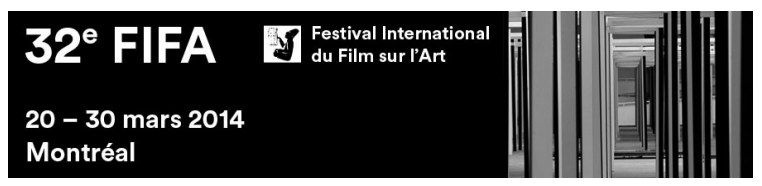

Pour cette $32^{\mathrm{e}}$ édition, René Rozon et son équipe ont réussi une impressionnante moisson : 266 films dont 40 appartiennent à la rubrique «musique», une année faste. Voici la recension de 11 d'entre eux.

\section{Films de la catégorie "musique" en compétition}

Cette année, le jury était composé de quatre réalisateurs de Suisse, de France et d'Acadie, sous la présidence de Danielle Sauvage, précédente directrice du Conseil des arts de Montréal. Ils ont eu à choisir entre $3^{8}$ films, dont huit portaient sur la musique, entièrement ou partiellement.

\section{L'autre Karajan, Autriche/Allemagne, Eric} Schulz, 2012, 52 minutes, allemand avec soustitres français

Prix ex cequo du meilleur film pour la télévision, ce film va directement au cour de la relation de Karajan avec le son. Le titre allemand Die Zweite Leben correspond d'ailleurs plus précisément au propos : il ne s'agit pas d'un autre Karajan, mais bien d'une seconde vie. On y scrute sa manière d'enregistrer, sa relation avec les ingénieurs de son, relation extrêmement proche, même s'ils ne lui obéissaient pas toujours... Il est tout à fait fascinant d'être témoin de son intérêt, de son enthousiasme pour le tout nouveau disque compact ou pour de nouvelles techniques d'enregistrement, en tirant parti, par exemple, d'une prise de son avec beaucoup de microphones unidirectionnels. En filmant de près les interprètes et ingénieurs en train d'écouter des extraits de film ou de conversations avec Karajan, le réalisateur réussit à capter sur leurs visages toute l'admiration et l'émotion que nous éprouvons au même moment. Brillant! Ainsi, les commentaires sur l'enregistrement par Günther Hermans des Variations pour orchestre de Schönberg donnent envie de courir les réécouter. De son côté, la violoniste Anne-Sophie Mutter démontre une compréhension intime de la vision de Karajan sur l'importance de l'ingénieur comme «âme» du disque. Et finalement, on imagine que le célèbre chef d'orchestre aurait sans doute voulu tout réenregistrer avec l'arrivée des nouvelles technologies numériques!

Une chaise pour un ange, Canada/Finlande, Raymond St-Jean, 2013, 74 minutes, français, anglais, finnois, avec sous-titres français

Comment traduire l'esthétique Shaker ${ }^{1}$ tout en l'expliquant, voilà le propos du réalisateur Raymond St-Jean, inspiré par la démarche du chorégraphe finnois Tero Saarinen. Le résultat est un film dont le cœur est la chorégraphie pour huit danseurs de Saarinen, magnifiquement éclairée et filmée en Finlande dans un ancien bâtiment animé du même esprit que ceux des bâtiments Shakers. La chorégraphie, comme le 


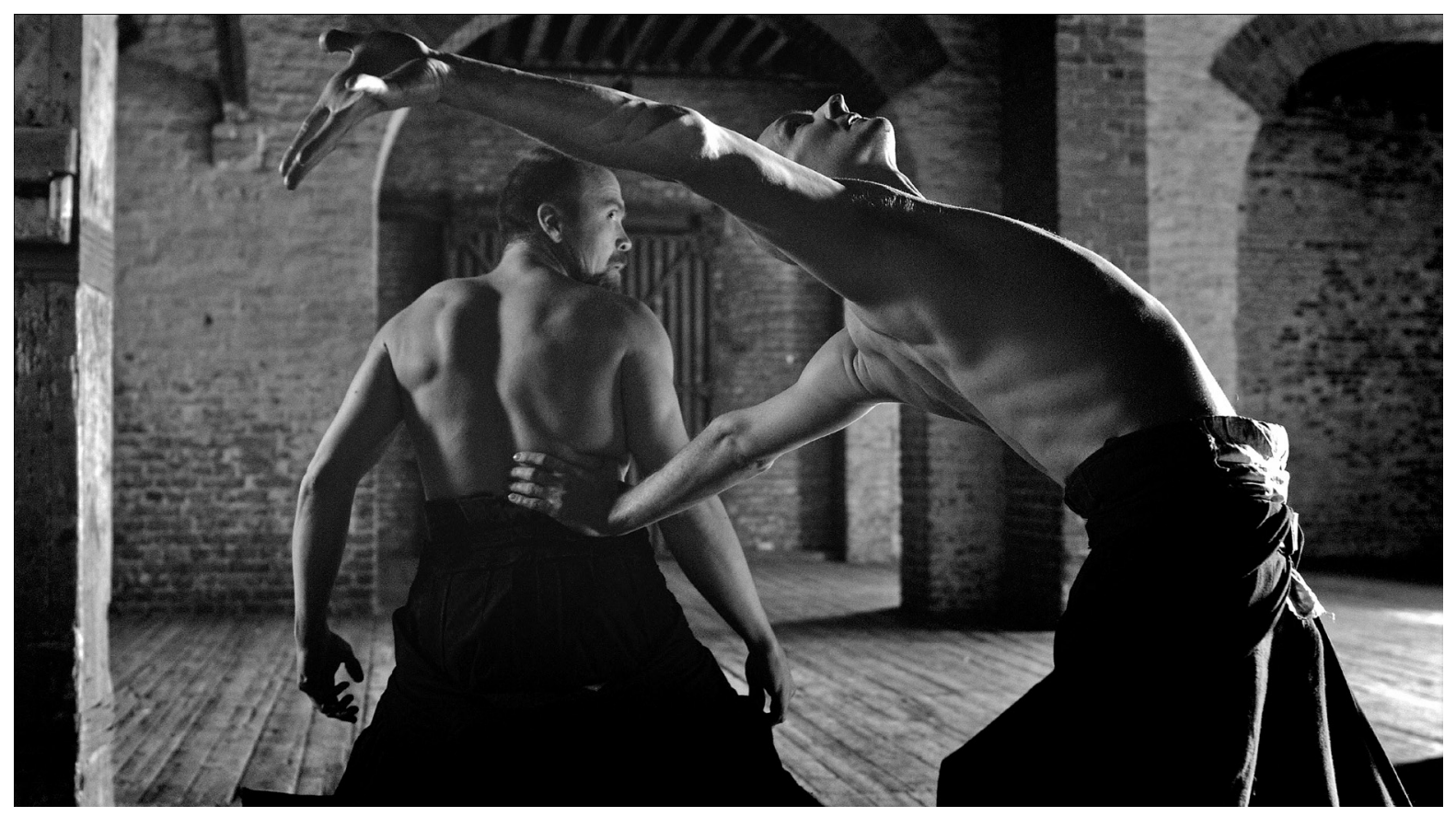

рното Image tirée du film Une chaise pour un ange de Raymond St-Jean.

film, sont alimentés par le design parfait d'un village Shaker, par l'explication de la musique Shaker par les directeurs de la Boston Camerata, Joel Cohen et Anne Azéma, et par les commentaires captivants d'un ébéniste sur les meubles Shaker. Et pour éclairer le sens de cette pureté fonctionnelle, un membre de la communauté exprime, en termes sobres, la simplicité de ces productions que nous qualifions d'artistiques. Récompensé par le Prix de la meilleure œuvre canadienne et par le Prix ICI ARTV pour la meilleure réalisation canadienne francophone, Une chaise pour un ange, est somptueux; un film d'art sur l'art, qui aurait mérité de remporter la palme du meilleur film.

Benjamin Britten - Peace and Conflict, RoyaumeUni, Tony Britten, 2013, 110 minutes, anglais

Film bien construit, bien filmé, avec une ligne éditoriale claire: explorer l'origine et l'incarnation de reconstituées de l'adolescence de Britten, présenté en jeune homme délicat, mais déterminé à être compositeur, et de documents d'archives, la démonstration est réussie. Dans cet univers presque exclusivement masculin, où la culture occupe une dimension centrale et cruciale, il était logique pour Britten d'exprimer ses opinions par la musique et son Requiem est sans doute son œuvre la plus explicite à cet égard.

Maurizio Pollini, de main de maître, France, Bruno Monsaingeon, 2013, 55 minutes, italien avec sous-titres français

Film classique et néanmoins intéressant sur le parcours de Pollini, à partir d'une entrevue en italien et en français, dans son bel appartement milanais, où on le voit étudier des partitions, sans son piano. Son enfance y est évoquée, de même que le concours Chopin gagné à 18 ans. Il raconte son amitié avec Claudio Abbado et Luigi Nono, son engagement politique et culturel, 
et fait l'amer constat que tous ces concerts populaires dans des usines n'ont donné aucune suite. À la fin du film, on le connaît un peu mieux, et on a fait le plein d'images d'archives. Mais il reste surtout l'impression d'assister à la satisfaction du réalisateur d'avoir mis la main sur Pollini, lui si avare d'entrevues.

\section{Dmitri Hvorostovsky: The Music and I, Nika Strizhka, 2012, 39 minutes, russe avec sous- titres anglais}

Contrairement à ce que le titre promet, ce film sans grandes qualités cinématographiques n’effleure même pas le rapport de la célèbre basse à la musique. On y voit un homme naïf, encore surpris de son talent, mais qui sait en profiter. Espérons que l'artiste n'est pas aussi superficiel que le film le laisse entendre. Totalement sous le charme, la cinéaste ne donne la parole qu'à la vedette. On le voit d'une limousine à l'autre, d'une représentation à l'autre, sans rapport réel à la musique - on pourrait faire le même film sur un footballeur. Certes, on devine une sincérité, mais rien n'est dévoilé d'une quelconque démarche artistique sérieuse, même si la voix est très belle.

\section{Quelques films de "musique" hors compétition}

\section{The Making of Fallujah: A New Chamber} Opera, Canada/États-Unis, John Bolton, 2012, 23 minutes, anglais avec sous-titres français

Genèse de la création d'un opéra thérapeutique sur l'expérience vécue par un soldat américain revenu blessé de la bataille de Fajullah en Iraq. On y entend le soldat, le mécène qui propose de faire de son histoire une œuvre d'art pour sensibiliser au syndrome posttraumatique, les producteurs du City Opera Vancouver et son chef, Charles Barber. La librettiste raconte son histoire et le compositeur canadien Tobin Stokes, son arrivée dans le projet. Difficile de juger de l'œuvre avec les quelques extraits entendus en version lecture.
La musique est dans la foulée de la plupart des opéras anglophones actuels, qu'ils soient néo-zélandais, américains ou canadiens. Le livret réaliste, exposant les tourments du personnage principal, exsude une atmosphère tragique semblable à celle du récent opéra Dead Man Walking de Jake Heggie. Reste à voir ce qu'une véritable production pourrait tirer de ce projet louable.

\section{Shikisou, Canada, Barry Doupé et Yota \\ Kobayashi, 2010-2012, 12 minutes, sans dialogue}

Film engendré par la musique plutôt que l'inverse habituel. Nous sommes dans le même type de démarche que celle du compositeur et vidéographe montréalais Jean Piché, avec beaucoup moins de succès cependant. La musique électroacoustique de Kobayashi, basée sur des sons de la nature, entraîne des variations de lumière et de couleurs sur un cercle, lui-même dans un rectangle. Charmé un moment, l'intérêt s'étiole dans la rigidité du cadre visuel et la lassitude de cette musique étonnamment datée du jeune compositeur canadien d'origine japonaise.

Textile de cordes, Canada, Nathalie Bujold, 2013, 1 minute 20 secondes, sans dialogue

Vidéo d'art trop brève, en forme de geste visuel et musical à partir d'images de la violoncelliste Isabelle Bozzini. Étrange que le générique fasse si peu de cas de la musique de Taylor Brook et que la description du film dans le catalogue l'évacue complètement...

\section{Herqueville, Canada, Pierre Hébert, 2007, 21 minutes, français avec sous-titres anglais}

Cette juxtaposition sensible de la musique de Fred Frith avec les dessins de Michelle Corbisier, les animations de Hébert et l'intégration ludique des poèmes de Serge Meurant est très réussie. La musique agit comme déclencheur de gestes graphiques animés et humanise ces rochers et ces paysages qui se passeraient bien des hommes. On est intrigué par des visions d'usine 
jusqu'à ce que le texte final nous l'explique: il s'agit d'une usine de transformation de déchets nucléaires. Il s'agit, comme le dit bien Hébert, d'une méditation sur la tragédie de ce lieu, et il le fait en utilisant sa propre sensibilité et celle des trois artistes. Chapeau.

\section{Harmonielehre, Canada, Michel D.t. Lam, 2013, 40 minutes, sans dialogue}

Expérience visuelle bien plus qu'auditive, cette projection en 360 degrés sur le dôme de la Société des arts technologiques (SAT) est une fantaisie visuelle répétitive et sans véritable fil conducteur, dans laquelle surgissent quelques bonnes trouvailles. La diffusion qui se veut immersive aplatit les dynamiques et finit par rendre insignifiante l'Harmonielehre de John Adams interprété par l'Orchestre symphonique de Montréal (OSM), dirigé par Kent Nagano. La référence au rêve d'Adams (un superpétrolier s'élançant de la baie de San Francisco) par lequel le film est introduit évacue complètement le titre de l'œuvre, emprunté au livre de Schoenberg, et à toutes les références historiques que Adams a introduites dans l'œuvre. Les rares spectateurs ont dû se demander qui étaient ces visages qu'on voyait apparaître (surtout Schoenberg) ou ces lettres manuscrites en allemand adressées à lui. Au-delà du bateau s'envolant près du Golden Gate de San Francisco, l'iconographie laisse une impression de fourre-tout: colonnes, paysages lunaires, formes colorées, caméra glissant dans la maison symphonique et au travers de l'orchestre, gros plans de musiciens. On sort de la salle en ayant à peine effleuré l'œuvre, distraits et étourdis par un visuel mouvant que l'on peine à raccorder à l'œuvre. Le plus beau reste l'affiche: un élégant graphisme coloré qui découpe le titre pour mieux en évacuer le sens.
In Between: Isang Yun, in North and South Korea, Allemagne, Maria Stodtmeier, 2013, 60 minutes, allemand avec sous-titres anglais

Film intéressant sur ce compositeur respecté par les deux Corées qu'il souhaitait tant réunir. Kidnappé par la Corée du Sud, torturé, condamné, libéré par des pressions internationales, il est devenu un citoyen allemand et passera la moitié de sa vie en Allemagne. Compositeur dodécaphoniste, sa musique a été adoptée par les deux Corées, qui ont chacune créé un institut portant son nom. Et lorsque l'on assiste à un concert en Corée du Nord, en 2008, où l'orchestre Isang Yun accompagne un soliste originaire du Sud, on se dit qu'Isang Yun a peut-être réalisé son souhait... Le film est surtout riche de rares images du Nord, à l'éducation et à la culture rigides. Quand Yun avait visité la Corée du Nord au début des années 1960, il avait trouvé le projet communiste porteur d'espoir, de là ses sympathies. Il l'aura payé cher, lui comme tout le peuple du Nord, quand le communisme a cédé le pas à la dictature complète.

Plusieurs autres films auraient mérité un commentaire, The Perfect American, par exemple, l'opéra de Philip Glass sur Walt Disney, ou ce vieux documentaire de Nigel Finch, My Way, véritable incursion musicologique dans les multiples incarnations de cette chanson de Claude François, adaptée par Paul Anka. La $32^{\mathrm{e}}$ édition du Festival International du Film sur l'Art méritait une fréquentation assidue, et les amateurs de musique y ont certainement trouvé leur compte, même si, selon nos goûts, la musique contemporaine n'y était pas suffisamment présente.

\footnotetext{
1. La communauté Shaker est une secte protestante implantée aux États-Unis au XVIII' siècle, aujourd'hui quasi éteinte, mais dont quelques villages ont été préservés. Ils ont développé un style de vie minimaliste qui se transposait dans des maisons et des meubles aux lignes épurées, qui sont considérées à l'origine du style scandinave de la moitié du $\mathrm{xx}^{\mathrm{e}}$ siècle et font maintenant partie de nombreuses collections de musées. Leurs chants sont moins connus, mais reflètent cette même volonté de simplicité.
} 